\title{
NAVIGARE NEL MEDIOEVO. ASPETTI FINANZIARI DELLE SQUADRE NAVALI: \\ IL CASO DELLA SPEDIZIONE PER LA \\ LIBERAZIONE dI MARIA dI SICILIA
}

Pinuccia Franca Simbula

Istituto sui Rapporti Italo-Iberici,

CNR, Cagliari (Italia)

"Senyor, no sol no em pens que galera ne altre vaixell gos anar sobre mar menys de guiatge del rei d'Arago, ne encara no solament galera ni lleny, mas no creu que negun peix gos alçar sobre mar si no porta un escut ab senyal del rey d'Arago"'.

Con queste eloquenti parole fatte pronunciare all'ammiraglio Roger de Lauria, il cronista Ramon Muntaner nei primi decenni del Trecento sintetizzava l'ambiziosa politica catalano-aragonese. E' probabile che, come ironicamente ricorda Hillgarth, tali parole strappassero il sorriso del conte di Foix, al quale erano dirette; ed è certamente vero che il brano tende a rappresentare un'immagine della monarchia ferma e sicura nella propria potenza. I sovrani catalano-aragonesi, nel tentativo di accreditare l'aggressiva politica internazionale, ricorsero anche a messaggi propagandistici, celebrativi ed intimidatori nello stesso tempo, affidandone la diffusione ad

\footnotetext{
'Bemat Desclot, Llibre del rey en Pere, cap. 166, a cura di F. SOLDEVILA, Les quatre grans Croniques, Barcelona, 1971, p. 577.
} 
un mezzo immortalante e divulgativo della grandenza delle cronache composte dalla storiografia di Corte nel basso medioevo ${ }^{2}$.

Davanti a tali affermazioni, specchio dell'impostazione politica della Corona, risultano interessanti le verifiche delle reali possibilità economiche, delle strutture e degli strumenti di conquista e di controllo disponibili nelle fasi dell'espansione mediterranea. Un'espansione che segno una decisa svolta negli equilibri politici, militari e commerciali del tempo sul piano internazionale, ma ebbe effetti e riflessi di enorme portata anche su quello interno. Effetti che condizionarono in modo determinante non solo gli aspetti finanziari ed economici, ma gli stessi rapporti tra l'autorità regia e le classi sociali, coinvolte in modo diretto o indiretto nella complessa macchina innescata da queste guerre di lunga durata. Del resto una molteplicità di fonti e di analisi storiografiche recenti hanno messo in luce su piú piani contraddizioni, fratture interne e limiti dell'espansione ${ }^{3}$.

Un buon esempio costituiscono in questo senso i risultati dei recenti studi -in buona parte ancora in corso- condotti da Manuel Sánchez Martínez sui tempi e sui modi di costruzione di un apparato di esazione fiscale e di controllo delle rendite della Corona, studi che contribuiscono a mettere in luce come la complessa macchina si sviluppi e si perfezioni in relazione al vorticoso aumento delle esigenze finanziarie; il che a partire dal principio del Trecento significo concretamente un aumento delle necessità militari determinate dalle campagne di conquista mediterranee ed in particolare dei regni insulari di Sardegna e Corsica e Sicilia ${ }^{4}$.

\footnotetext{
${ }^{2}$ J. N. HILLGARTH, El problema del imperio catalano-aragonès (1229-1327), "Anuario de Estudios Medievales" (da qui "AEM") 10 (1980), p.157.

${ }^{3} \mathrm{M}$. TANGHERONI, Il regnum Sardiniae et Corsicae nell'espansione mediterranea della Corona d'Aragona. Aspetti economici, in La Corona d'Aragona in Italia (secc. XIII-XVIII), "Atti del XIV Congresso di Storia della Corona d'Aragona", Alghero 1990, Sassari 1993, pp. 49-88; A. SANTAMarla, Precisaciones sobre la expansión marítima de la Corona de Aragón, "Anales de la Corona de Aragón de la Universidad de Alicante" 8 (1990-1991), pp. 187-285; J. M. SALRACH, Balance crítico y perspectivas de la producción historiográfica sobre historia medieval catalano-balear en la década 1975-1986, "Studia Historica: Historia Medieval" 6 (1988), pp. 95-139.

${ }^{4}$ M.T. FERRER I MALLOL, El patrimoni reial $i$ la recuperació dels senyorius jurisdicionals en els estats catalano-aragonesos a la fi del segle XIV, "AEM", 7 (1970-71), pp. 351-491; M. SANCHI:Z MARTINEZ, Después de Aidu de Turdu (1347): los sucesos de Cerderia y sus repercusiones en el patrimonio real, "XIV Congresso di Storia della Corona d'Aragona", Sassari-Alghero, 1990, pre-print, Addenda, pp. 110-135; IDEM, La fiscalidad real en Cataluria (siglo XIV), "AEM", 22 (1992), pp. 341-376; di grande interesse anche il recente volume a cura del medesimo M. SANCHEZ MARIINEZ, Estudios sobre renta, fiscalidad y finanzas en la Cataluña bajomedieval, Barcelona, 1993.
} 
L'osservazione della normativa sugli armamenti navali e dei metodi di finanziamento è una ulteriore spia delle necessita finanziarie della Corona. In particolare e la connotazione strutturale dell'organizzazione delle squadre navali, espressa dalla stessa normativa, a mettere l'accento sulla necessità di sistemi di reperimento alternativi ai finanziamenti erogati dalle casse statali per mantenere in navigazione le imbarcazioni delle squadre regie ed ottenere squadre piú offensive nonché il controllo delle acque nel Mediterraneo occidentale. E su queste basi sembra crescere l'inserimento di capitani corsari che, sotto le bandiere catalano aragonesi, cosí fittamente popolarono il Mediterraneo tra Tre e Quattrocento. L'importanza e la consistenza della guerra di corsa ed il suo ruolo come strumento di conquista della Corona sono dimostrate da numerosi studi condotti soprattutto dalla Ferrer che ha messo in luce come, nel contesto delle difficoltà economiche, venisse sapientemente utilizzata per sostenere la politica bellica ${ }^{5}$.

Nelle Ordenanzas Navales Pietro IV d'Aragona, oltre a precisare competenze, ruoli, modalità di arruolamento dell'equipaggio, salari e norme per le armate navali, aveva regolamentato anche la figura del clavario, un ufficiale di nomina regia con competenze amministrative, appositamente imbarcato sulle navi armate con finanziamenti statali. Al clavario spettava, oltre che amministrare le somme versate dalla Corte, anche il compito di reebe tot lo guany que nostre Senyor dard, incamerare cioe i proventi dei bottini di guerra, i beni di proprietà nemica di cui le navi della flotta entravano in possesso de bona guerra che, una volta inventariati, potevano essere venduti. Gli introiti dovevano essere utilizzati per le spese necessarie a mantenere l'imbarcazione in navigazione; eventuali eccedenze dovevano

${ }^{5}$ M.T. FERRER I MAIJOL, Els corsaris castellans $i$ la companya de Pere Niño al Mediterrani. Docuinents sobre El Victorial, "AEM" 5, 1968, pp. 265-338; IDEM, Productes del comerç calcalano-portugues segons una reclamació per pirateria (1408), "Miscel•lània de Textos Medievals", 6 (1992), pp. 137-163; IDI:M, Dos registres de "l'Officium Maris" de Gènova (1402-1403, 1408-1410), "Alti del I Congresso Storico Liguria-Catalogna", Bordighera, 1974, pp. 248-348; IDEM, La conquista di Sardegna e la guerra di corsa nel Mediterraneo, in "I Catalani in Sardegna", Cagliari, 1985, pp. 35-40; IDEM, Barcelona $i$ la política inediterrànea catalcina: el Parlainent de 1400-1401, "XIV Congr. di Storia della Corona d'Aragona", Sassari-Alghero 1990 (in corso di stampa); IDEM, El cors català contra Genova segons una reclamació de 1370, in "Sardegna Mediterraneo e Atlantico tra Medioevo ed Eta Modema. Studi Storici in memoria del prof. Alberto Boscolo" a cura di L. d'Arienzo, Roma, 1993, pp. 269-280. Oltre ai lavori della Ferrer i Mallol, numerusi riferimenti in G. MELONI, Genova e Alagona all'epoca di Pietro il Cerimonioso, Padova, 1971-1982, 3 voll.; E. PUTZULU, Pirati e corsari nei inari della Sardegna durante la prima metà del secolo XV, "IV Congreso de Historia de la Corona de Aragón", Mallorca, 1959 e P.F. SimBULA, Corsari e pirati nei mari di Sardegna, Cagliari, 1993. 
essere versate alla Corte ${ }^{6}$. Su questi "danneggiamenti" inflitti ai nemici e sui relativi guadagni si fondava la possibilità di un consistente alleggerimento delle spese per le casse regie. Ma non solo. In caso di necessità era infatti possibile che si potessero effettuare dei dirottamenti di imbarcazioni mercantili per procurare vettovaglie agli eserciti impegnati sulla terraferma, per gli equipaggi delle galere ecc. Le spedizioni regie, per sostenere gli alti costi, facevano esplicito affidamento sulle integrazioni dei bottini che le squadre avrebbero razziato durante la navigazione ${ }^{7}$. Anche in questo caso la pratica era antica. I bottini erano vitali per le imbarcazioni della flotta: erano beni rapidamente convertibili in moneta ed evitavano pericolosi malcontenti a bordo nei ritardi dei pagamenti da parte della Corte ${ }^{8}$.

Oltre ai libri contabili delle galere delle armate reali, una testimonianza precisa è quella offerta dai registri dei notaments comuns del Maestro Razionale. Qui, sistematicamente, a partire dalla seconda meta del Trecento, soprattutto relativamente alle operazioni militari per la conquista del regno di Sardegna, sono raccolti i documenti che testimoniano da parte dei sovrani il riconoscimento dei debiti ai mercanti danneggiati dalle unità della flotta ${ }^{9}$.

\footnotetext{
'A. de CApMany y de Monpalau, Ordenanzas de las Armadas Navales de la Corona de Aragón, Madrid, 1787, p. 120; A. GARCLA I SANS, Historia della marina catalana, Bareelona, 1977, pp. 87-88.

${ }^{7}$ A. de CAPMANY, cit., capp. VII-VIII, pp. 47-49. Un clavario doveva essere presente su ogni imbarcazione o in ogni squadra armata con finanziamenti pubblici, comprese -nel caso di contributi finanziari regi- quelle dei corsari che affiancavano le unita della flotta nelle operazioni condotte dalla Corona.

${ }^{8}$ Un esempio è quello di Berenguer Morey che, per mancanza di denaro e di vettovaglie, decise di appostarsi nelle Bocche di Bonifacio per attaccare le imbarcazioni di passaggio. Archivo de la Corona de Aragón (in seguito ACA), Maestro Racional (in seguito MR), reg. 2308, fol. 6 r, cit. in P.F. SimBULA, Corsari e pirati, cit., p. 48. A questo proposito sono significative le richieste di risarcimento presentate da Genova a Pietro IV, nel 1370, relative per la maggior parte agli anni 1366-69. Il re si impegnava a rifondere i danni causati a 28 imbarcazioni dai propri sudditi, tra i quali i capitani delle galee regie. I nomi sotto accusa erano quelli di Francesc d'Averso, Olf da Procida, Berenguer Morey, Berenguer Sespujades. M.T. FERRER I MALLOL, La conquista, cit. pp. 35-36; M. DEL TREPPO, I mercanti catalani e l'espansione della Corona d'Aragona nel secolo XV, Napoli, 1972, pp. 418-423; M.T. FERRER I MALLOL i A. GARCIA I SANS, Asseguranges $i$ Canvis Marítims Medievals a Barcelona, Barcelona, 1983, I, pp. 211-213.

${ }^{9}$ ACA, MR, regg. 786-793 (anni 1372-1400). In questi registri sono compresi esclusivamente $\mathrm{i}$ riferimenti a risarcimenti riconosciuti dal sovrano per requisizioni di beni a sudditi alleati o mercanti di nazionalita amica; un piú ampio panorama dei danneggiamenti inflitti si ritrova, oltre che in diverse serie di registri di Cancelleria (Marcarum, Sardiniae, Sigilli Secreti), nei libri dei clavari delle imbarcazioni della flotta regia. Relativamete agli incidenti nei mari di Sardegna cfr. P.F. SIMBULA, Corsari e pirati, cit., Tabella VI.
} 
Attaccare i sudditi della Corona, oltre che i nemici, ha dunque una legittimità dettata dalle necessita belliche a cui si pone rimedio con la formula del risarcimento. Principio che sarà applicato largamente in Sardegna dalle città regie e dagli stessi ufficiali, ma pratica usuale già tra Due e Trecento, come numerosi passi della Cronica del Muntaner confermano ampiamente ${ }^{10}$. I dirottamenti, inseriti tra le voci che componevano le entrate del regno sardo o delle galere che pattugliavano le acque, venivano addebitati al sovrano. Agli aggrediti si rilasciava una dichiarazione nella quale si riportava la composizione e il valore dei beni sequestrati. Nel caso in cui venisse presentata la richiesta di risarcimento, si doveva allegare la carta debitoria rilasciata dagli ufficiali regi, unitamente a quanto fosse necessario per dimostrare la propietà dei beni per i quali si chiedeva l'indennizzo. La stima, inserita nei libri degli amministratori del regno, serviva come verifica per effettuare il risarcimento. Sostanzialmente sembra una sorta di anticipo, di prestito ottenuto con la forza, per il quale veniva computato dalla Corte anche un indennizzo di $2 \mathrm{~s}$. per lira ${ }^{11}$.

La prassi dei dirottamenti, confondendosi con le operazioni di guerra di corsa, si presenta costante durante tutta la seconda metà del Trecento nella guerra di conquista del regno di Sardegna e nelle operazioni per l'affermazione del dominio diretto in Sicilia, avviate a partire dagli ultimi anni di regno di Pietro IV d'Aragona ${ }^{12}$.

Apparentemente, dunque, sembra che, nel caso di appropriamento di beni di sudditi o di alleati e neutrali, il problema si risolvesse per la vittima con un risarcimento e un'indennizzo per $i$ danni causati e per la Corona con una dilazione nel reperimento dei fondi. In realtà la Corona si

\footnotetext{
${ }^{10}$ Uno dei sistemi utilizzati per sostenere le unità della flotta in navigazione era il ricorso alle requisizioni dei carichi. La prassi, per la seconda metà del Trecento, era stata sottolineata e documentata diversi anni fa da Ciro Manca il quale evidenziava per il regno di Sardegna come le navi venissero forzatamente dirottate nei porti per approvigionare le città assediate. C. MANCA, Fonti ed orientamenti per la storia economica della Sardegna aragonese, Padova, 1967, p. 44. R. MUNTANER, Cronica, a cura di F. SOlDEVILA, Les quatre, cit., cfr. l'emblematico cap. CXCIV, pp. 840-842.

"C. ManCa, Fonti, cit., p. 44.

${ }^{12}$ Relativamente alla Sicilia emblematica e la concessione di un feudo in Sandegna agli eredi del corsaro Johan de Castrillo che aveva a lungo servito il re Martino. ACA, Cancilleria (in seguito C), reg. 2398, f. 28v-30. Per un inquadramento della corea nel regno di Sicilia efr. H. BRESC, Politique et société en Sicile, XIle-XVe siecles, Great Yarmouth (Norfolk), 1990, $e$ in particolare l'XI saggio. Di grande interesse anche la periodizzazione delle fasi della corse proposta dallo studioso; per il regno di Sardegna, P.F. SimBULA, Corsari e pirari, cit.
} 
garantiva rapidamente gli introiti e i privati non sempre ottenevano la rifusione dei danni.

Un esempio è offerto da un intervento militare nel regno di Sicilia: la spedizione per la liberazione di Maria di Sicilia tra il 1380 ed il 1381, nella quale troviamo aggregati, a fianco agli ufficiali regi, corsari di professione e mercanti. Si tratta di un caso per il quale i documenti consentono di seguire nei dettagli gli sviluppi e le azioni, grazie a quanto rimasto del libro dell'amministrazione di bordo del clavario e alle corrispondenti registrazioni nei libri del razionale di Corte, dove si ritrovano le partite relative alle entrate, alle uscite e soprattutto ai risarcimenti di cui la Corona dovette farsi carico per l'attività della squadra ${ }^{13}$.

\section{LA SPEDIZIONE IN SiCILIA}

Nel gennaio del 1379 Guglielmo Raimondo III di Moncada, signore di Augusta, riusciva a sottrarre alla custodia di Artale de Alagona la giovane Maria, regina di Sicilia. Successivo passo fu quello di offrire la preda a Pietro IV d'Aragona il quale con la sua molt cara Maria avrebbe avuto il controllo diretto del regno. Le manovre per l'inserimento diretto catalanoaragonese in Sicilia cominciavano con un colpo di mano. Una trattativa, quella condotta tra Pietro IV e il Moncada, nella quale sarebbero confluite le ambizioni del nobile siciliano, frustrato dalla forzata marginalità in quegli anni dai vertici del potere in Sicilia e le aspirazioni sul regno da parte catalano-aragonese $\mathrm{e}^{14}$.

Le trattative tra Pietro IV e il Moncada, recatosi personalmente a Corte, furono lente ed improntate a una prudenza che evidenzia sospetti e diffidenze reciproche. A Corte per trattare si trovava anche un altro partitario aragonese, Enrico Rosso ${ }^{15}$.

\footnotetext{
${ }^{13} \mathrm{Si}$ tratta del registro della clavaria di Berenguer de Guardiola, clavario della squadra inviata per detta liberazione. Il registro, frammentario, consente di ricostruire in buona parte la spedizione. Conservato nella sezione del Maestro Razionale dell'ACA, è indicato con la segnatura reg. 2317.

${ }^{14}$ P.F. SIMBULA, Corsari e pirati, cit., cap. VI.

${ }^{15}$ Sul nuolo di Enrico Rosso nelle vicende legate alla regina Maria e al Moncada cfr. M.R. LO FORTE SCIRPO, Cronaca di un rapimento e di un riscatto, in "Atti del XIV Congr. di Storia della Corona d'Aragona", Sassari-Alghero, 1990 (in corso di stampa) e la documentazione citata.
} 
La situazione per il sovrano catalano aragonese non si prospettava semplice: le difficoltà finanziarie della Corona, come ricordava qualche tempo dopo al padre anche l'infante Martino, erano manifeste "a tots vostres sotsmeses, et plagues a Deu que no ho fos a altres estranys de diverses partides del mon" ${ }^{16}$. Il reperimento di fondi e la ricerca dell'adeguata ricompensa fece trascorrere diversi mesi. Gli accordi vennero raggiunti nell'estate del $1380^{17}$. Allora partirono i preparativi per la spedizione che avrebbe preso in consegna la giovane regina. Nei porti di Barcellona, Valenza e Maiorca furono armate tre galere. Al comando erano rispettivamente Miquel de Gurrea, Jacme Rigolf e i corsari maiorchini Arnau Aymar con Pere Agullo; capitani della squadra Eximén Pérez de Arenós e Roger de Moncada ${ }^{18}$. Clavario era Berenguer de Guardiola il quale, aiutato da dei sostituti imbarcati sulle galee di Valenza e di Maiorca, doveva amministrare finanziariamente la squadra. Oltre alle tre galere, come imbarcazione da trasporto per i soldati e le armi, nonché come appoggio nelle operazioni militari, veniva inviata anche la nave di en Gelats, un armatore catalano spesso ricordato nelle fonti ${ }^{19}$.

Nelle loro mani Guglielmo Raimondo di Moncada si era impegnato a consegnare la regina $\mathrm{Maria}^{20}$. Compito non facile e tanto piú complesso quanto piú si allungavano i tempi: le fortezze siciliane di Augusta e di Licata, dove Maria veniva tenuta prigioniera, erano sorvegliate strettamente quando non tenute d'assedio ${ }^{21}$.

Il dettagliato resoconto della spedizione della squadra navale si è in buona parte conservato e consente di osservare l'attività durante l'arco di

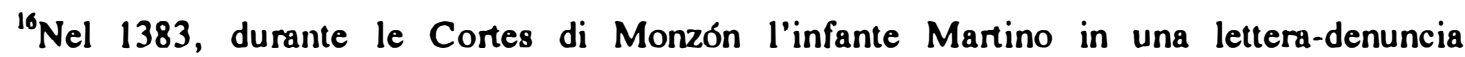
ricordava pubblicamente al padre come nonostante le iniziative e le forti esazioni fiscali "vostre patrimoni és diminuït en tant que és quasi a extrem de periclitar". Cfr. I. BaIGES I JARDI-A. RUBió I RODOn-E. VAREla RODRIGUEz, Cort General de Montsó, Barcelona, 1992, p. 100; M.T. FERRER I MALLOL, El patrimoni reial, cit.

${ }^{17}$ M.R. LO FORTE SCIRPO, Cronaca di un rapimento, cit.

${ }^{18}$ E. Perez de Arenós, nobile valenzano, cra nel 1380 camerlengo dell'infante (ACA, C, reg. 2069, f. 105).

${ }^{19}$ ACA, MR, reg. 2317, f. 4 r; MR, reg. 789 , f. 69 r. e v.

${ }^{20}$ Il documento e ricordato da M.R. LO FORTE SCIRPO, Cronaca di un rapimento, cit., pp. 556-557.

${ }^{21}$ Qualche contributo finanziario è registrato in uscita dalla tesoreria gia nella primavera: il 4 aprile 1380 Guglielmo Raimondo de Moncada ricevette 4000 fiorini d'oro d'Aragona "per affari segreti della Corte". Anticipo per il conte o contributo per sostenere le difese? La notizia $d$ in ACA, MR, reg. 788, f. $267 \mathrm{v}$.
} 
tempo della permanenza in mare. Il libro del clavario offre molte informazioni e il valore dei dati contenuti è di grande interesse su un duplice piano: in primo luogo sulla struttura finanziaria delle flotte regie catalanoaragonesi, sulla loro composizione e sulla necessita delle aggressioni; inoltre è rivelatore di correnti commerciali mediterranee spesso minori e meno documentate.

Della squadra approntata per la Sicilia facevano parte la galea La Vittoria, al comando di Michel de Gurrea, La Sant Jaume, guidata da Jacme Rigolf e la Sant Salvador e Santa Clara di proprieta dei corsari maiorchini Arnau Aymar e Pere Agullo ${ }^{22}$. Alle galere del valenzano Jacme Rigolf e dei maiorchini Arnau Aymar e Pere Agullo, che mettevano a disposizione le loro imbarcazioni, veniva versata, oltre alle somme per pagare i salari agli imbarcati, le armi, le vettovaglie il "calament" 23.

Il reperimento dei fondi allungo i tempi di allestimento della squadra che solo al principio dell'inverno del 1380, partl dai porti di armamento $^{24}$.

La difficoltà maggiore era quella di reperire le somme di danaro necessarie $e^{25}$. Gli stessi ordini impartiti alla squadra in partenza indica la carenza di liquidita della Corte. Esplicitamente veniva consentito e richiesto ai capitani delle galere per mantenere in navigazione la squadra "que faessen guerra a tots aquells que ells volrien e presessen bens axi de sotsmeses del dit senyor com d'altres, axi en aquella manera que per los dits nobles (Roger de Moncada e Eximén Perez de Arenos) lus seria manat e ordonat". Con un altro documento, i patroni erano autorizzati a prendere beni "de sotsmeses del dit senyor e d'altres tots bens, aytant empero com fossen necessaris per sosteniment dels castells quels dits nobles tendrien en Sicilia e de les gents d'armes e sou de les galeres" ${ }^{26}$. Sostanzialmente l'autorizza-

\footnotetext{
${ }^{22}$ P.F. SIMBULA, Corsari e pirati, cit., cap. V.

${ }^{23} \mathrm{ACA}, \mathrm{MR}, \mathrm{rcg} .789$, f. $68 \mathrm{r}$ e v. Il calament consisteva in un indennizzo che il prestatore pagava all'entità prestatrice coıne indennizzo per la perdita di valore sofferta dai beni dati in uso. A. GARCIA I SANS-N. COLL. I JULȦ, Galeres inercants catalanes del segles $X V V$ i XV, Barcelona, 1994, pp. 278-281.

${ }^{24}$ I contratti con $i$ capitani furono firmati tra l'estate e l'autunno; la partenze della galea La Vittoria e della Sant Salvador avvenne nella seconda metà di dicembre. ACA, C, reg. 1405, ff. 10-11 v.; 22 v-23 v.; MR, reg. 789, ff. 67 r-68 v.

${ }^{25}$ M.R. LO FORTE SCIRPO, Cronaca di un rapimenso, cit., pp. 556-559 ricorda la lentezza dei preparativi della squadra.
}

${ }^{26} \mathrm{ACA}, \mathrm{MR}$, reg. 2317, f. 6. 
zione regia faceva si che de bona guerra o per necessità chiunque potesse essere attaccato.

Al patrono in caso fosse di nazionalità catalana, amica o neutrale era rilasciata la carta debitoria con l'inventario ed il relativo valore dei beni sequestrati, del risarcimento dei quali, come prassi consolidata, si sarebbe fatta carico la Corte.

L'attività della squadra è estremamente significativa. L'ordine regio fu applicato alla lettera: tra il mese di dicembre 1380 ed il maggio del 1381 , arco di tempo nel quale rimase in navigazione, furono attaccate circa 35-40 imbarcazioni, tra quelle fermate per i carichi e quelle assaltate per recuperare uomini da mettere al remo: fiorentini, genovesi, maltesi, siciliani, corsi, provenzali, pugliesi, cagliaritani e catalani furono in numerosi casi spogliati di tutto, ben oltre le necessità di vettovagliamento di equipaggi e truppe ${ }^{27}$.

Le registrazioni del Guardiola tracciano il percorso della squadra. La rotta seguita costeggiava la Catalogna settentrionale, risaliva verso la Provenza per poi tagliare verso la Corsica e la Sardegna ed infine raggiungere la Sicilia dove le galere, impegnate a pattugliare i mari, trascorsero i mesi fino all'estate del 1381.

Le fasi piú intense delle aggressioni si concentrarono in due momenti: subito dopo la partenza tra le coste del Rossiglione e la Provenza e soprattutto sulla costa meridionale della Sicilia nei mesi primaverili ${ }^{28}$.

Durante le prime settimane di navigazione, nelle acque di Port Vendres furono attaccati 5 liuti barcellonesi muniti di salvacondotto e una nave genovese che per il riscatto dei panni e delle spezie trasportate furono costretti a versare 3.000 fiorini d'oro d'Aragona. Nelle vicinanze, a Sant Feliu de Guixols era stato preso un altro liuto ${ }^{29}$.

Nelle vicinanze di Marsiglia furono invece fermate la cocca di Thomas Sala di Barcellona con merci fiorentine e una galeazza di Aigues Mortes che rientrava dal Levante, sulla quale erano imbarcati alcuni fattori

\footnotetext{
${ }^{27}$ Furono allaceule almeno 33 imbariarini per ciarichi, scali e vomini; alcune non precisubili solo per reperire unmin da mellere al remo e lurono eflelluale due razie a terra durante i corcanalatges clifelluali " Capo Passero, qui lurono presi degli schiavi. ACA, MR, reg. 2317, Il. 48 r, 55 r. Per la callura delle imburcivioni vedi labella (p. 506).

${ }^{28}$ Furono elfelluali 7 altaceti tra Port Vendres e Marsiglia, incentre il resto degli episodi ricordati si sviluppò tra le coste campanc, la Sicilia e la Calabria.

${ }^{29}$ ACA, MR, reg. 2317, i. $56 \mathrm{v}$.
} 
fiorentini diretti a Montpellier ai quali fu presa una modesta somma in monete d'oro ${ }^{30}$.

A Gaeta fu intercettata la cocca di Pere Garraf di Tortosa il quale dovette pagare un riscatto per evitare il sequestro delle merci. La galea di Aymar e Agulló giunse per prima in Sardegna dove sosto a Castell de Caller, portandovi per la vendita le prime imbarcazioni catturate ${ }^{31}$. Dalla galea dei corsari maiorchini, la prima a giungere in Sardegna, fu preso anche un panfilo di Antich Paschal e di Johan de Casellas di Barcellona che per fortuna que havien hauda era stato spinto in quelle acque. Il carico, grano, vino, sardine e panni era stato inviato da mercanti marsigliesi ai connazionali impegnati ad Alghero nella pesca del corallo ${ }^{32}$.

Il grosso delle catture è pero concentrato in Sicilia. Circa una ventina di imbarcazioni furono intercettate lungo le coste della Sicilia, alcune in acque calabresi. Capo Passero, Augusta, Catania, Siracusa e Reggio risultano i settori piú redditizi e indicano comunque un maggior stazionamento della flotta nella parte merionale sicula, tra Augusta e Licata, dove si sviluppano le manovre militari per prendere in consegna la regina Maria.

Qui furono intercettati i carichi di maggior rilievo: le grandi navi che caricavano grano in Sicilia, quelle che tornavano dal Levante con sete e spezie; i piccoli legni maltesi impegnati nel commercio del cotone ${ }^{33}$.

L'obiettivo della squadra è realizzare rapidamente i bottini per trasformarli in denaro per l'acquisto di vettovaglie e armi. Quando le vendite nei porti non sembravano equivalere al giusto valore o non si presentavano comunque convenienti, il clavario trasforma le merci in strumento di pagamento in natura dei salari per gli equipaggi, per i noli corrisposti ad alcune delle imbarcazioni attaccate e in qualche caso anche per spese delle navi di sostegno. Un caso è quello della tarida di Nichola Prea di Bari catturata nello stretto di Messina, davanti a Reggio. Sulla nave oltre a grano, orzo, olio e mandorle furono trovati IIII barrils de moneda veya de

\footnotetext{
${ }^{30}$ ACA, MR, reg. 2317 , ff. 6 v., 9 r. e v.

${ }^{31}$ ACA, MR, reg. 2317, ff. 22 r. 24 v., 34 r. Pere Garraf di Tortosa aveva subito qualche anno prima un altro attacco. Nel 1368 un suo legno era stato dirottato ad Alghero con un carico di 90 salme di grano, misura di Mazzara. Il valore del carico ammontava a 213 ll. e 1 8. barcellonese. ACA, MR, reg. 785 , f. $1 \mathrm{r}$.

${ }^{32} \mathrm{ACA}, \mathrm{MR}$, reg. 2317 , ff. 22 r. e v.

${ }^{33}$ ACA, MR, reg. 2317 , ff. 46 v. -56 v.
} 
pitxols de Napols del peso di 10 q. 10 libbre e 8 once barcellonesi, di proprietà di mercanti di Reggio e di Firenze. Inizialmente fu portata ad Augusta dove verosimilmente furono ripartite o vendute le vettovaglie; per le monete si ritenne opportuno tentare a Napoli. Qui, dopo vani tentativi "per ço com no la us volien ben comprar en Napols ne non sen daven tant qui muntan 761 florins d'Arago" i pitxols furono dati in paga all'equipaggio. Solo una parte fu venduta al napoletano miçer Gandolf per un totale di $218 \mathrm{f}$. d'oro d'Aragona 7 s. $11 \mathrm{~d}^{34}$.

Gran parte del carico di seta grezza sequestrata alla nave castigliana di Ferrando Guerra di Sant'Ander, fermata in Sicilia a Capo Passero, fu utilizzata per pagare il soldo alla ciurma. Anche la farina, il grano, il vino, le spezie furono spesso utilizzati in mancanza di contanti ${ }^{35}$.

I carichi piú consistenti messi in vendita furono immessi nel porto di Cagliari dove vino e vettovaglie erano ben accette viste le necessità di rifornimenti della citta ${ }^{36}$. Il resto, come un carico di seta trattenuto dal clavario, fu utilizzato prevalentemente per acquistare scorte alimentari o, come si è detto, effettuare pagamenti in natura. Sono registrati anche piccoli incanti nei porti siciliani e in Calabria; a Girgenti dove "per ço com no si acustuma de res vendre en encant públich" una barca ed un modesto carico di un genovese furono venduti attraverso un sensale a Francisco Xicho, botiguer $^{37}$.

Le piazze maggiormente utilizzate per la vendita furono -oltre a Cagliari- Augusta, Licata, Reggio e Napoli.

Il valore dei bottini dichiarati o consegnati dai patroni al clavario oscilla tra le 6 lire barcellonesi prese ad un giovane fattore fiorentino lungo le coste della Provenza e le 4.157 lire barcellonesi della nau castigliana

${ }^{34}$ ACA, MR, reg. 2317, ff 50 r., 54 r.

${ }^{35}$ Alle galere della squadra le vettovaglie venivano segnate tra le distribuzioni di viveri; alla nave di sostegno di Matheu Gelats in qualche caso fu data parte di carichi sequestrati come paga per i servigi che rendeva; è il caso delle 4 carregues di pepe prese dalla nave di Guillem Nicholau di Tortosa che rientrava da Alessandria con un carico di spezie. ACA, MR, reg. 789 , ff. 73 v. 74 r.

${ }^{36}$ P.F. Simbula, Corsari e pirati, cit.

${ }^{37}$ Oltre allo scafo furono messe in vendita 12 mans di carta, una colta di maglia, qualche capo di abbigliamento per un totale di $92 \mathrm{ll} .9 \mathrm{~s} .1 \mathrm{~d}$. barcellonesi. "I oratori... per ço com valia fort poch e no era cosa que dagués vendra" e pochi panni furono regalati allo scrivano della galea. ACA, MR, reg. 2317 , f. 47 r. 
carica di seta di proprietà di mercanti costantinopolitani e veneziani, diretta a Maiorca ${ }^{38}$.

La tipologia prevalente delle imbarcazioni fermate, oltre a barche, liuti e panfili, è quella delle navi ${ }^{39}$. Ne furono attaccate ben quattro, una delle quali carica di cotone e grano fu trovata da Aymar e Agullo "desemperada en les mars de Saragossa que no-y havia negun" e fu da loro e da Guglielmo Raimondo di Moncada che no volch res fer trattenuta; tanto che il clavario annoto come si fosse fermamente quanto inutilmente opposto ${ }^{40}$; un problema, questo, che dovette spesso affrontare, stando a quanto annota con una certa desolazione in piú punti del suo libro ${ }^{41}$ : 50 sacchi di farina furono presi forclvolment dai corsari maiorchini Arnau Aymar e Pere Agulló da una barca catanese; un bell arnès de cavaller complit fu preso da Francesch Sent Vicens, del loro equipaggio; ancora Aymar e Agullo con il conte di Augusta catturarono una nave carica di cotone e grano ${ }^{42}$. Gli stessi capitani della flotta si impossessarono di merci che inviarono direttamente alle loro case ${ }^{43}$. Ugualmente il Guardiola si dovette arrendere davanti al sequestro della nave del genovese Costantino Adorno che aveva caricato grano a Catania, da parte di Maria di Sicilia, che su consiglio del Moncada la trattenne en nom seu ... de bona guerra ${ }^{44}$.

I carichi piú frequentemente colpiti, oltre a quelli di area catalanoaragonese, sono siciliani, provenzali, fiorentini e soprattutto genovesi. I danni, in particolare per questi ultimi, sono di una certa entita; oltre alla nave dell'Adorno, fermata ad Augusta, nel libro della clavaria del Guardiola sono esplicitamente ricordati diversi sequestri di panni, vino, grano, olio, denaro e altro ${ }^{45}$.

\footnotetext{
${ }^{38} \mathrm{ACA}, \mathrm{MR}$, reg. 2317, if 43 r. -44 r. II bollino in alcuni casi poteva essere anche molto alto: il valore dei earichi di harche o di piecole imbarcirioni come pantili, cafiuree, leuts, si aggirava tra le 100 e le 500 lire; talvolta la ciallura di grosse imbarciasoni frullava anche mole

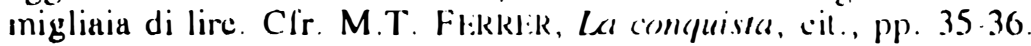

${ }^{39}$ In prevalenza le vittime dellat syuadra sono barche (10) e liuti (8), ancle se non maneano imbareazioni di notevoli dimension e eapacitia di carico, come navi (5), cocche (3) e panfili (4). Cfr. Tabella, p. 506.

${ }^{40}$ ACA, MR, reg. 2317, f. 56 r.

${ }^{41} \mathrm{ACA}, \mathrm{MR}$, reg. 2317, l. 6r. $68 \mathrm{r}$.

${ }^{42}$ ACA, MR, reg. 2317, if. 48 r., 54 v. $-55-r ., 65$ v. -67 r

${ }^{43} \mathrm{ACA}, \mathrm{MR}$, reg. 2317, f. $67 \mathrm{r}$.

${ }^{44} \mathrm{ACA}, \mathrm{MR}, \mathrm{reg} .2317$, ff. 50 v., 54 r., 55 r

${ }^{45} \mathrm{Cf}$ r. Tahclla (p. 506).
} 
Piú numerosi ancora risultano i sequestri di imbarcazioni e di carichi catalani, per i quali i mercanti avevano maggiori possibilità di ottenere i risarcimenti; anche se dopo complesse e lunghe procedure. Vediamone un esempio. Nell'aprile del 1381 era stata catturata a Capo Passero la nave carica di seta di cui era patrono Ferrando Guerra di Sant'Ander. Mercante della nave era il cavaliere di Costantinopoli Johan Lescari. Il valore, già ricordato, superava le 4.157 lire barcellonesi. Una parte dei costals di seta era stata data in accomandita al maiorchino Arnau Oliver e a due fattori del Lescari. Parte del carico era di Berenguer Mira di Perpignano, parte di Johan Andreu di Maiorca. L'Oliver fu il primo ad avviare la pratica di risarcimento che il re gli riconobbe il 3 ottobre di quello stesso anno. Gli venivano concessi $1081 \mathrm{ll} .7 \mathrm{~s} .8 \mathrm{~d}$. barcellonesi delle quali 720 ll. $16 \mathrm{~s} .8$ d. per il valore della seta (a 40 ss. barcellonesi per libbra); 110 ll. barcellonesi per "messions e despeses quel dita Arnau havia fetes siguent la cort del senyor rey e del senyor duch per demanar lo dit deute"; 152 II. $5 \mathrm{~s}$. per 203 ducati che aveva investito nella seta del Lescari; 98 11. 6 s. come indennizzo per il mancato guadagno corrisposto nella misura di $2 \mathrm{~s}$. per lira. Il tutto gli era stato riconosciuto dopo la presentazione dell'albard scritto di pugno dal clavario Berenguer de Guardiola ${ }^{46}$ e di una carta publica del notaio di Maiorca Bonanat Sala, datata 6 giugno 1381, nella quale i fattori del Lescari riconoscevano all'Oliver la proprietà dei quantitativi dichiarati alla Corte.

Piú complessa l'operazione del mercante Berenguer Mira che ottenne il riconoscimento del debito nel marzo 1382. Oltre all'albard del Guardiola il Mira presento una serie di carte che servivano a dimostrare le complesse operazioni di prestiti e di cessioni di crediti effettuate da diversi mercati su somme dovute dal Lescari che andavano a beneficio del $\mathrm{Mi}$ $\mathrm{ra}^{47}$. Nell'ottobre fu infine riconosciuto a Johan Andreu, mercante di Maiorca, il debito di 476 ll. $6 \mathrm{~s}$. barcellonesi per i quantitativi di seta che gli erano stati dati in accomandita dal veneziano Johan Sobranço a Modo, dove

\footnotetext{
${ }^{46} \mathrm{Nel}$ quale indicava di aver ricevuto la detta seta e di averla impiegata per pagare gli equipaggi della flotta $\mathrm{e}$ il nolo del trasporto dei soldati e dei balestrieri dalla Catalogna in Sicilia. ACA, MR, reg. 2317, ff. 249 v.-250 v.
}

${ }^{47}$ ACA, MR, reg. 789 , ff. 1 r. -2 v. 
la nave aveva effettuato il carico ${ }^{48}$. Mediamente le spese sostenute per ottenere il riconoscimento del debito si aggirarono tra il $9 \%$ e il $15 \%{ }^{49}$.

Il riconoscimento dei crediti da parte della Corte non significava, come è intuibile, l'immediato pagamento, per il quale i danneggiati avrebbero dovuto ancora attendere. Per la maggior parte delle imbarcazioni battente bandiera catalana, indipendentemente dalla nazionalità del carico e dal successivo risarcimento, risulta il pagamentro del nolo ai patroni ${ }^{50}$.

Anche il commercio di cabotaggio e quello a breve distanza risulta in queste operazioni pregiudicato dalla squadra catalana. E' certamente un commercio minore, quantitativamente e qualitativamente: sono le barche e i liuti siciliani, calabresi e maltesi con poche salme di grano, farina e altre vettovaglie, quantitativi di vino e cotone a cadere preda dei catalani ${ }^{51}$. Mentre per i fiorentini, considerati nemici, e quindi i loro beni bottini "de bona guerra" non vi erano possibilità di risarcimento, per i mercanti genovesi rimaneva la via delle proteste ufficiali e le richieste di indennizzo da inoltrare attraverso il Comune alla Corte catalana.

Per i mercanti catalani e i neutrali o amici le carte debitorie rilasciate davano diritto al risarcimento; tuttavia per i piccoli mercanti, per quanti erano impegnati nel commercio di cabotaggio, era spesso oneroso anticipare le somme per l'istruzione delle pratiche, motivo per cui le perdite, seppure decisamente minori, incidevano maggiormente. Per i patroni, in quei casi spesso proprietari delle merci, generalmente si trovano i versamenti dei noli, mentre non compaiono richieste ufficiali di risarcimento, costose e lunghe da avviare per i modesti sequestri come quelli subiti ${ }^{52}$.

Un altro aspetto riguardante i naviganti assaliti e quello della costrizione al remo. Per la galea La Vittoria, il clavario ricorda una quindicina di uomini forzatamente arruolati, tra cui un pescatore napoletano e un

\footnotetext{
${ }^{48} \mathrm{ACA}, \mathrm{MR}, \mathrm{reg} .789$, f. $46 \mathrm{r}$.

${ }^{49}$ ACA, MR, reg. 788 , ff. 249 v. -250 v; reg. 789, ff. 1 .r-2 v., 46 r.

${ }^{50} \mathrm{Cfr}$. nota 52.

${ }^{51}$ In particolare i carichi dei fiorentini sono esplicitamente considerati de bona guerra. Cfr. ACA, MR, reg. 2317, f. 96 r. Sui fiorentini nella Corona d'Aragona cfr. M.T. FERRER, Els italians a terres catalanes, "AEM", 10 (1980), pp. 453-458.

52Sono ricordati i pagamenti effettuati a Berthomeu Rocha, Antich Pasqual e Johan de Casellas, Thomas Sala, Nardo de Capallina, Guillem Nicholau, per il nolo di 5 barche siciliane. ACA, MR, reg. 2317, ff. 51 v., 98 r. e v., 102 r., 104 r., 105 r., 107 r.
} 
lombardo che aveva fatto parte di un panfilo bruciato dalla squadra a Frigols $^{53}$.

Il libro della clavaria del Guardiola suggerisce anche qualche considerazione sulla composizione delle somme impiegate per la flotta. Complessivamente furono spese $13.515 \mathrm{ll} .16 \mathrm{~s} .3 \mathrm{~d}$. barcellonesi e al momento del pareggio dei conti al Guardiola dovevano essere versate ancore 17411.10 d. barcellonesi. Sempre questo documento registra in entrata 13.341 11. 15 s. 4 d. barcellonesi delle quali solo 2.186 ll. 18 s. $10 \mathrm{~d}$. barcellonesi, equivalenti circa al $16,5 \%$ furono versati dalla Corte, mentre il resto fu incamerato con requisizioni e assalti ad imbarcazioni di passaggio dalle tre unità della squadra.

L'affare per la Corte si dimostrava certamente vantaggioso. Anche tenendo conto delle somme di cui si faceva carico restituire -all'incirca 4.600 lire barcellonesi- restava piú o meno un $40 \%$ dei bottini incamerato direttamente dal clavario che se non apportava consistenti introiti, aveva il duplice vantaggio di consentire la permanenza in mare della squadra e di dilazionare il momento in cui si sarebhero dovuti reperire $i$ fondi per $i$ risarcimenti che avvenivano comunque in maniera parziale ${ }^{54}$. Oltre ai benefici derivati dal realizzo dei bottini per la Corte, da questo sistema traevano dei vantaggi anche i patroni e $\mathrm{i}$ capitani delle galere incamerando bottini a titolo personale, impossibili da quantificare, ma intuibili dalle ricordate proteste del clavario.

\footnotetext{
${ }^{53}$ Il Guardiola tra gli altri ricorda: Johan Pera, preso su una cocca davanti a Sciacca; Alfonso de Cardona, Pcre Bosch, Francesch Morell di Maiorca e Pero Ferrandis di Siviglia presi su un panfilo a Tropea; Johan de Concha preso su una barea armata; Barthomeu Johan preso da un liuto a La Lena; Xico Xeraulo di Siracusa, Cola di Mazzara, Nardo del Goy, Simo Bartxaya e Pino Damasqueno (Malta), che avevano fatto parte dell'equipaggio corsaro della barca di 22 remi di Nicola Bocar di Trupani catturata nelle acque di Siracusa. ACA, MR, reg. 2317, ff. 165 v., 175 r., 177 r., 195 v. -197 r.

${ }^{4}$ Con una certa approssimazione, dai calcoli latti altraverso $\mathrm{i}$ registri dei notaments comuns del inaestro razionale, scmbra che solo una parte dei danneggiati avviasse la pratica per il riconoscimento dei danni ed il conseguente risarcimento. ACA, MR, regg. 788 e 789.
} 


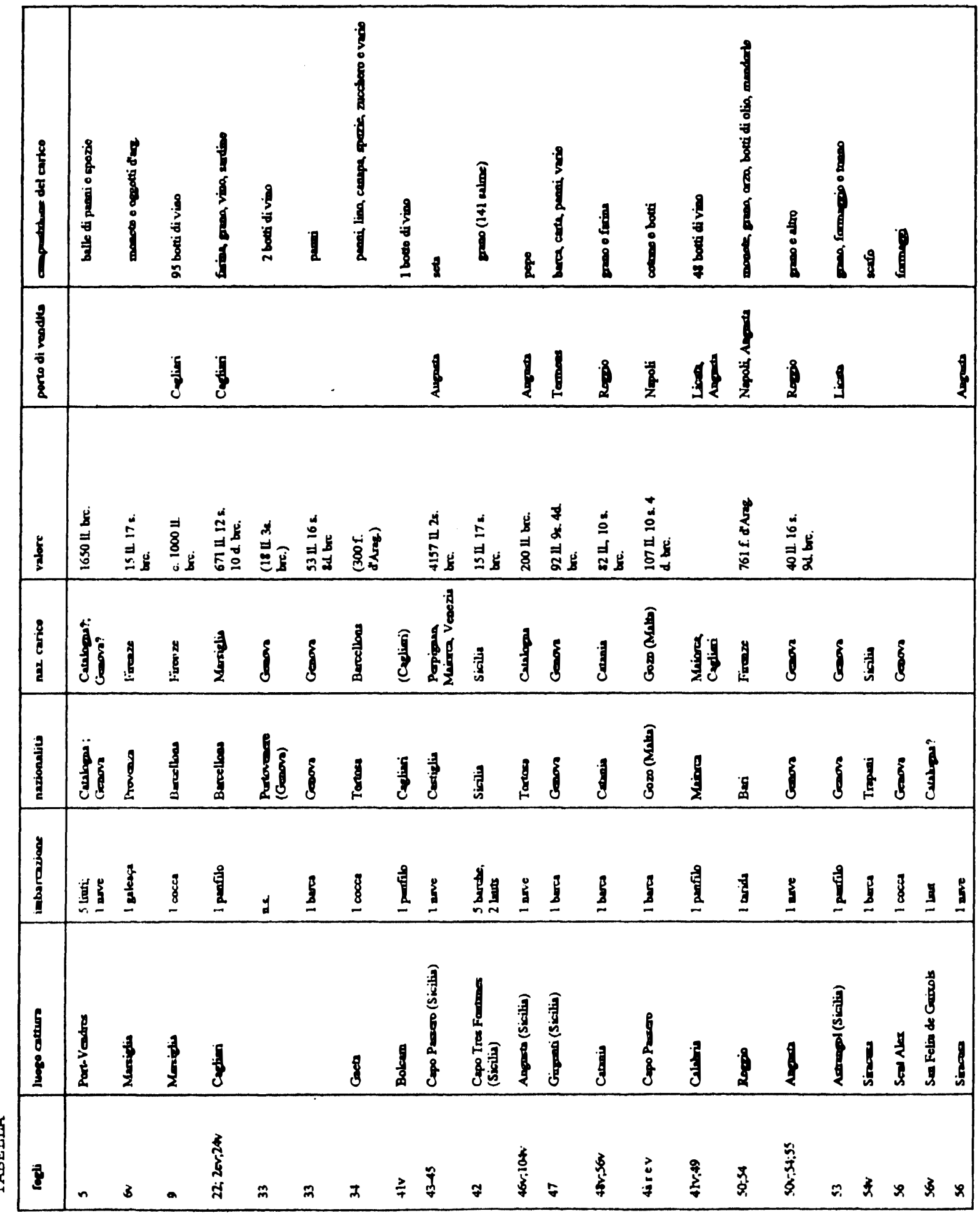




\section{RÉSUMÉ}

En considérant l'importance de la guerre de course dans l'expansion Catalane-Aragonese entre le $\mathrm{XIV}^{e}$ et le $\mathrm{XV}^{e}$ siècles, quelques opérations militaires conduites et projetées par la Couronne à la fin du XIV ${ }^{\circ}$ siècle pendant la guerre pour la conquête du royaume de Sardaigne et du royaume de Sicile ont été examinés. Les registres comptables restés nous ont permis l'examen des données économiques et financiers, la compositions des escadres navales, l'insertion de capitaines corsaires et leurs activités. En particulier on a analysé la comptabilité de l'escadre navale envoyée en Sicile entre 1379 et 1380 pour libérer la reine Marie, l'activité de la flotte et l'utilisation du butin pour amortir les grands frais de la guerre.

\section{SUMMARY}

By considering the importance of corsairs on the Catalan-Aragonese expansion between the XIVth and the XVth centuries, this article examines several military operations planned and carried out by the Crown at the end of the XIVth century, during the war to conquer the kingdom of Sardinia and the kingdom of Sicily. By analysing the left bookkeeping, the economic and financial data were evaluated as well as the composition of the squadrons, the recruit of corsair captains and their activity. In particular, we analyse the bookkeeping of the squadron sent to Sicily (1379-1380) to free the queen Maria, as well as its activity and the importance of booty in sustaining the war high costs. 